\title{
A natureza da pragmática: percurso teórico em um piscar de olhos ${ }^{1}$
}

\author{
The nature of pragmatics: a theoretical review in a blink of an eye \\ Ana Maria T. Ibaños \\ Jorge Campos da Costa \\ Pontifícia Universidade Católica do Rio Grande do Sul - Porto Alegre, RS, Brasil
}

$\diamond$

\begin{abstract}
Resumo: O propósito deste trabalho é apresentar aspectos gerais sobre o desenvolvimento da pragmática, desde suas origens e discussões estritamente teóricas, no que concerne às pragmáticas e suas vertentes. De fato, tal disciplina pode ser caracterizada de várias maneiras, especialmente como subárea da Linguística Pura, ou como forma de abordagem de caráter instrumental, em que se identificam contextos para as diversas situações de uso da linguagem. Dada essa complexidade que envolve a Pragmática, parece justa a pretensão de nosso trabalho ao ser voltado para os fundamentos, conceitos básicos e debates típicos. Isso vai na direção de familiarizar e permitir compreender as questões motivadoras de pesquisa que cercam uma multiplicidade de problemas relevantes na área.
\end{abstract}

Palavras-chave: Pragmática; Conceitos pragmáticos; Pragmática clássica; Pragmática contemporânea

\begin{abstract}
The purpose of this work is to present general aspects of the development of pragmatics, from their origins and strictly theoretical discussions, with regard to pragmatics and their strands. In fact, such discipline may be characterized in many ways, especially as a subarea of Pure Linguistics, or as an instrumental approach, in which contexts are identified for the various situations of language usage. Given this complexity that involves pragmatics, it seems fair to the pretension of our work when being focused on the fundamental, basic concepts and typical debates. This goes in the direction of familiarizing and allowing to understand the motivating issues of research that surround a multitude of relevant problems in the area.
\end{abstract}

Keywords: Pragmatics; Pragmatic concepts; Classical pragmatics; Contemporary pragmatics

\section{Introdução}

Escrever sobre um recorte histórico-teórico da Pragmática requer fazer uma escolha entre as possibilidades de divisão, levando-se em consideração o aspecto mais relevante para o trabalho. Levinson (1983) considera a pragmática como o significado menos as condições de verdade; em (2000) considera a Pragmática com duas perspectivas: numa, ela participa da constituição da proposição para que adquira condições-de-verdade; na outra, é a identificação de implicaturas modelo griceano. Korta e Perry (2015) optam por classificar a pragmática dentro do escopo de pragmática near-side (próxima)

\footnotetext{
O título de nosso texto se baseia ostensivamente nos textos de Allwood A bird's eye view of pragmatics, e de Monawar Evolução da Faculdade da Linguagem, Gramática Universal, Princípios e Parâmetros e Modelo Computacional de Turing em uma Casca de Amendoim.
}

e far-side (distante), já iniciando o seu estudo pelos trabalhos de Grice e Austin. O caminho histórico teórico aqui adotado leva em consideração esses autores, mas se divide em Período Clássico e Contemporâneo, levando em consideração a história da definição de pragmática e seu objeto. Por se tratar de um percurso teórico "em um piscar de olhos", obviamente, nem todos os aspectos poderão ser contemplados. Mas o que se segue, preenche o propósito do artigo.

\section{Pragmática - período clássico $x$ período contemporâneo}

A divisão em período clássico e contemporâneo, embora discutível, tem como propósito evidenciar o fato de que o caminho da pragmática segue o caminho de filósofos da lógica e filósofos da mente. Por clássico, entendemos o 
período do final do século XIX até fins da década de 50 e início da década de 60, quando a pragmática foi definida de forma não homogênea por Peirce (1897), Morris (1938), Carnap (1939) e Bar-Hillel (1954)². Por contemporâneo, a pragmática inaugurada por Grice (1957) com Meaning e Austin (1962) com seu livro How to do things with words, cujo interesse era (e ainda é) discutir o além do dito, até os teóricos atuais, que se afiliam a estudos de pragmática empírica, pragmática de corpus, pragmática experimental, teórica ou histórica. ${ }^{3,4}$ Iniciamos pelo momento clássico, ou, no que podemos chamar de "definições de pragmática e estabelecimento de seu objeto.

\section{Período Clássico}

Peirce, no final do século XIX, refere-se à relação dos signos com seus interpretantes no seu trabalho sobre Semiótica, e parece que Charles Morris (1938) se baseou nele, quando propôs a definição tripartida de semiótica em sintaxe, semântica e pragmática. De acordo com o autor:

It is a sufficiently accurate characterization of pragmatics to say that it deals with the biotic aspects of semiosis, that is, with all psychological, biological and sociological phenomena which occur in the functioning os signs (MORRIS, 1938, p. 108). ${ }^{5}$

Contudo, o compromisso de Morris com uma filosofia behaviorista e lógico-empiricista dá à definição de pragmática um escopo por demais amplo, chamado de concepção maximalista. Este primeiro uso do termo (amplo) cobre sociolinguística, psicolinguística, neurolinguística, entre outros, e não serve para nossos propósitos de um estudo dentro de uma concepção mais afinada, num primeiro momento, com a definição linguística.

Necessário dizer, no entanto, como Allwood (1978, p. 145) ressalta, em Morris (1938), sintaxe era o estudo mais abstrato dos signos, semântica mais concreto, incluindo tanto sintaxe quanto o estudo da denotação sem o uso, e pragmática seria todo o escopo do estudo da linguagem, incluindo sintaxe e semântica. Isso muda em Morris no seu texto Signs, language and behavior (1946), quando a semântica se torna o estudo da significação em

\footnotetext{
2 De acordo com Costa (1982), Frege (1898) e Wittgenstein (1953) tiveram uma grande intuição dos fenômenos pragmáticos, mas não chegaram a acusar a existência teórica de disciplina.

3 Ao contestar Russell (1905), Strawson (1950) também acusou a existência da Pragmática ao retomar o fenômeno da pressuposição, definido por Frege (1892/1978).

4 Cf. Perna, Cristina, Goldnadel, Marcos e Molsing, Karina. Pragmáticas - vertentes contemporâneas. Porto Alegre: EDIPUCRS, 2016.

5 A caracterização da Pragmática é suficientemente acurada para se dizer que ela lida com os aspectos bióticos da semiose, ou seja, todos os fenômenos sociológicos, biológicos e psicológicos que ocorrem no funcionamento dos signos .(tradução dos autores do texto)
}

todos os modos de significar e pragmática o estudo da origem, uso e efeitos dos signos.

Dentro da Filosofia Analítica, o termo pragmática sofre um estreitamento de escopo; Carnap (1942) o utiliza tanto para um ramo da ciência, como para o estudo da linguagem natural. Ele adicionou à tricotomia de Morris a noção de investigação de uma semiótica descritiva e uma semiótica pura. Uma investigação pura define o número de conceitos necessários para um sistema de signos; enquanto que uma investigação descritiva implica o estudo de sistemas reais de signos, isto é, um estudo descritivo de uma pragmática que lidará com questões como intenção, enunciado e crença, todos no nível abstrato que fazem referência a um agente. Nas palavras de Carnap (1942, p.9)

If in an investigation explicit reference is made to the speaker, or, to put it in more general terms, to the user of a language, then we assign it to the field of pragmatics. (...) If we abstract from the user of the language and analyze only the expressions and their designata, we are in the field of semantics. And if, finally, we abstract from the designata also and analyze only the relations between expressions, we are in (logical) syntax. ${ }^{6}$

De acordo com Costa (2008, p. 23):

O que caracteriza essa fase, sem dúvida, é o fato de que os teóricos como Morris ou Carnap compreenderam a necessidade de investigações que sistematizassem os fenômenos ligados ao uso da linguagem, antes de estabelecer, com clareza, os limites desse fenômeno e o aparato conceptual dessa investigação.

Bar-Hillel (1954) refere-se a essa distinção de pragmática descritiva e pura, sugerindo o que hoje chamamos de pragmática formal. Ele define pragmática como o estudo das línguas naturais e artificiais que contenham termos indexicais. Seu texto clássico Indexical Expressions foi uma as contribuições formadoras da pragmática contemporânea ${ }^{7}$. Seu trabalho chamou atenção ao fato de que expressões indexicais são ubíquas na linguagem natural e criam um problema para a Lógica. ${ }^{8}$ Em suas palavras:

Even very superficial investigation into the linguistic habits of users of ordinary language will reveal that

\footnotetext{
6 Se numa investigação é feita referência explícita ao falante, ou, de maneira mais geral, ao usuário de uma língua, então, designamos isso ao campo da pragmática. (...) se abstrairmos do usuário da língua e analisarmos somente as expressões e suas designata, estamos no campo da semântica. E se, finalmente, também abstraímos da designata e analisamos somente as relações entre as expressões, estamos na sintaxe (lógica).

7 Gazdar (1979) opõe-se a Bar-Hillel, uma vez que todas as línguas naturais possuem indexicais; pouco uso eles oferecem aos linguistas. Cf. Pullum and Gazdar, 1982.

8 Cf. Cummings, L. The Routledge Pragmatics Encyclopedia.
} 
there are strong variations in the degree of dependence of linguistic expressions on the pragmatic context of their production. Whereas, for instance, the sentence (1) Ice floats on water will be understood for almost every grown-up normal English speaker, [...] (2) It's raining $[\ldots]$ will be fully grasped only by those who know the place and the time of its production (BARHILLLEL, 1945, p. 359). ${ }^{9}$

Retomando, as sentenças como (1) e (2) abaixo traduzidas, mais a sentença (3):

(1) O gelo flutua sobre a água.

(2) Está chovendo.

(3) Estou com fome.

representam três tipos de sentenças. A sentença (1) pode ser perfeitamente entendida e avaliada; a sentença (2), para ser avaliada em toda sua extensão, necessita-se saber o lugar e momento de sua produção (seu enunciado); e a (3) exige saber-se quem a pronunciou, além do momento de enunciado. Para Bar Hillel, (1) é representativa de asserção e (2) e (3) são sentenças indiciais.

Wittgenstein em seu trabalho Investigações Filosóficas, cujo tópico poderíamos dizer que é a comunicação, trabalha com a noção de pragmática como a relação entre linguagem e usuários e estabelece um paradigma importante para estudos posteriores na sua Teoria dos jogos da linguagem: o significado é o uso:

"For a large class of cases of the employment of the word 'meaning' - though not for all — this word can be explained in this way: the meaning of a word is its use in the language" (WITTGENSTEIN, 2009, p. 43). ${ }^{10}$

Segundo Wittgenstein, há diversas atividades que chamamos de jogo. Mas não há algo constante chamado jogo. Jogar cartas, futebol, xadrez, vídeo-game, etc., são coisas muito diferentes. $\mathrm{O}$ que há são semelhanças de família. Usa-se a linguagem para promessas, ameaças, piadas, diálogos, informações, representação de fatos, entre $n$ coisas. O significado de uma palavra é o que se faz com ela em tais jogos. Nem objetos nem conceitos constituem o significado das palavras. A linguagem são jogos compartilhados publicamente. Não há exatamente linguagem privada.

\footnotetext{
9 Mesmo uma investigação muito superficial dos hábitos linguísticos de usuários da linguagem ordinária revelarão que existem fortes variações no grau de dependência das expressões linguísticas no contexto pragmático de sua produção. Enquanto, por exemplo, a sentença (1) O gelo flutua sobre a água será compreendida por quase todos os falantes adultos de inglês, [...] (2) Está chovendo [...] será totalmente entendida por aqueles que sabem o lugar e hora de sua produção.

10 Para um grande número de casos do emprego da palavra 'significado'embora não para todos - esta palavra pode ser explicada desta forma: o significado de uma palavra é o seu uso na linguagem.
}

Embora Wittgenstein não tenha discutido a disciplina Pragmática, sem dúvida alguma, o seu conceito de "jogos de linguagem" é uma das origens epistemológicas das teorias contemporâneas sobre o sentido das palavras (COSTA, 1982).

Até aqui, apresentamos o que convencionamos chamar de momento clássico da história da pragmática. Embora de maneira sucinta, parece-nos o suficiente para a visualização de parte do caminho histórico-teórico da disciplina. O Quadro 1 resume essa jornada teórica.

Quadro 1. Período clássico da história da Pragmática

\begin{tabular}{|l|l|l|}
\hline Frege & $\begin{array}{l}\text { Problema da pressuposição } \\
\text { e contexto }\end{array}$ & Investigação lógica \\
\hline Peirce & $\begin{array}{l}\text { Relação dos signos e } \\
\text { interpretantes }\end{array}$ & Investigação semiótica \\
\hline Morris & $\begin{array}{l}\text { Relação dos signos e } \\
\text { usuários }\end{array}$ & $\begin{array}{l}\text { Pragmática como área da } \\
\text { semiótica }\end{array}$ \\
\hline Carnap & $\begin{array}{l}\text { Semiótica descritiva e } \\
\text { pura }\end{array}$ & $\begin{array}{l}\text { Investigação lógico- } \\
\text { matemática }\end{array}$ \\
\hline Bar-Hillel & Expressões indiciais & Objeto da pragmática \\
\hline Wittgenstein & Jogos de linguagem & investigação filosófica \\
\hline
\end{tabular}

Partimos, então, para o segundo período:

\subsection{Período Contemporâneo}

Assumimos para este trabalho, a definição de pragmática que já está intimamente relacionada à questão linguística, ou seja, pragmática como subteoria linguística que investiga o significado na interface entre a sentença e o enunciado, como estudada por filósofos da linguagem como, Grice, Austin e Searle, entre outros.

Grice $(1957,1967 / 1975)$ estabeleceu o ponto de partida de uma verdadeira revolução copernicana na história das disciplinas da significação. Com Meaning e Logic and Conversation, ele praticamente estabeleceu a distinção entre duas diferentes e complementares disciplinas, a Semântica e a Pragmática. A Semântica, considerando o que foi dito, e a Pragmática, aquilo que foi implicado. Ele também entendeu que, normalmente, para que o que foi dito seja compreendido, dependemos de informações pragmáticas que não são apenas implicaturas. Um nome, por exemplo, depende de que sua referência seja estabelecida; um pronome, da mesma maneira. O básico, em Grice, foi a sua vontade teórica de compatibilizar os estudos formais e os informais.

Grice (1957) distingue no processo de comunicação, as noções de transferência de informação incidental e comunicação, propriamente dita, através do que ele chama de significado natural (como em nuvens pretas significam chuva) do significado não-natural, ou significado-nn ,equivalente à noção de comunicação intencional. Para o autor, 
(4) S meant-nn $z$ by uttering $U$ if and only if:

(i) $\mathrm{S}$ intended $\mathrm{U}$ to cause some effect $\mathrm{z}$ in recipient $\mathrm{H}$

(ii) $\mathrm{S}$ intended (i) to be achieved simply by $\mathrm{H}$ recognizing that intention (i) ${ }^{11}$

Conforme Levinson (1983, p. 16), esta definição essencialmente postula que a comunicação consiste na intenção comunicador (S) fazer com que o ouvinte (H) pense ou faça algo, uma vez que ele reconheça que o comunicador está tentando isso. O diálogo, peça comunicativa mais típica, caracteriza-se como significado ao nível do que é dito e ao nível do que é inferido.

Já em Logic and Conversation (1967/1975), estabelece um sistema conceitual altamente eficaz para explicar questões que envolvem o problema da significação em linguagem natural. Conforme Costa (1982, p.47),

a preocupação central de Grice era encontrar uma forma de descrever e explicar os efeitos de sentido que vão além do que é dito. Em última análise, como é possível que um enunciado signifique mais do que literalmente expresso. Deve haver algum tipo de regra que permita a um falante (A) transmitir algo além da frase e a um ouvinte (B) entender esta informação extra.

Em seu exemplo clássico, aqui traduzido em $(5)^{12}$,

(5) A e B conversam sobre um amigo mútuo, C, que agora está trabalhando em um banco. A pergunta a $\mathrm{B}$ sobre $\mathrm{C}$ e seu emprego; B responde: Oh, muito bem, eu acho; ele gosta de seus colegas e ainda não foi preso.

Grice introduz a noção de implicatura conversacional e apresenta sua Teoria das Implicaturas baseada num Princípio de Cooperação mais um conjunto de quatro máximas: quantidade, qualidade, relação e modo, a saber: ${ }^{13}$

(6) a) Máxima de Qualidade: não diga algo que você acredita ser falso e que não possa fornecer evidência adequada;

b) Máxima de Quantidade: torne sua contribuição tão informativa quanto é requerida e não dê mais informações do que o necessário;

c) Máxima da Relação: seja relevante;

d) Máxima de Modo: seja breve e ordenado, evitando obscuridade e ambiguidade.

\footnotetext{
${ }^{11} \mathrm{O}$ falante $\mathrm{S}$ não-naturalmente significou $\mathrm{Z}$ ao enunciar $\mathrm{U}$ se e somente se: (i) $\mathrm{S}$ pretendeu que $\mathrm{U}$ causasse algum efeito $\mathrm{z}$ no ouvinte $\mathrm{H}$; (ii) $\mathrm{S}$ pretendeu que (i) fosse atingido simplesmente pelo fato de $\mathrm{H}$ reconhecer a intenção de (i)

12 Grice,H.P. Logic and Conversation, 1967, p. 43

13 Cf. Costa, J.A Teoria Inferencial das Implicaturas: descrição do modelo clássico de Grice. Letras de Hoje, Porto Alegre, v. 44, n. 3, p. 12-17, jul./set. 2009
}

Em outras palavras: seja informativo como convém, diga a verdade, seja relevante e seja adequado na expressão. Quando o locutor aparentemente viola as máximas, ele pode ter intenção de gerar inferências. Com elas, pode-se transmitir mais do que o que é dito. São as implicaturas, inferências na interface semântico/ pragmática. De acordo com Grice, quando dois indivíduos estão dialogando, existem leis implícitas que governam o ato comunicativo. $\mathrm{O}$ autor, de certa forma, acredita que essas regras do ato comunicativo talvez tenham sido aprendidas concomitantemente à aquisição da linguagem, de tal forma que um falante competente de uma língua qualquer também conhece os efeitos de sentido que uma mensagem nessa língua pode adquirir pela ação das regras do jogo comunicacional a que está submetido.

Austin (1962), Searle $(1969,1975,1983)$ em seus trabalhos de filosofia da linguística desenvolvem a chamada Teoria dos Atos de fala que, como um meio de investigação pragmática, foi usada efetivamente tanto para estudos de aquisição de primeira como de segunda língua. De acordo com a Teoria de Atos de Fala, os falantes realizam atos ilocucionários ao produzirem sentenças. Um ato ilocucionário é uma função particular da linguagem realizada por um enunciado. Ou seja, através de seus enunciados, os falantes transmitem intenções comunicativas, tais como, pedidos, desculpas, promessas, conselhos, etc.

Em sua teoria, Austin dividiu os Atos de Fala em três tipos principais:

(7) a) Ato Locucionário: a produção de um enunciado que pode ser classificado por suas características fonéticas, gramaticais e lexicais até o significado da sentença. Também é o desempenho de um ato que pode ser classificado por seu conteúdo (o ato rético). De acordo com Austin,

If I promise that I'll be home for dinner and then promise that I'll work late, my actions are instances of two different locutionary acts: one with the content that I'll be home for dinner, and one with the content that I'll work late (1962, p. 94-98). ${ }^{14}$

b) Ato Ilocucionário: um ato classificado não apenas pelo seu conteúdo - como os atos locucionários - mas por sua força (prometer, aconselhar, ameaçar, etc.).

If I promise that I'll be home for dinner and later state that I'll be home for dinner, my actions are instances of the same locutionary act: both actions involve the content that I'll be home for dinner. However, my actions are instances of different illocutionary acts:

\footnotetext{
14 Se eu prometer que eu estarei em casa para o jantar e depois prometer que trabalharei até tarde, minhas ações são instanciações de dois atos locucionários distintos: um com o conteúdo de que estarei em casa para jantar, e outro com o conteúdo de que trabalharei até tarde.
} 
one has the force of a promise, while the other has the force of a statement (1962, p. 98-101). ${ }^{15}$

c) Ato Perlocucionário: ato classificado por seus efeitos sobre os sentimentos, pensamentos ou ações da audiência.

O exemplo dado por Austin que ilustra a noção dos atos de fala está representado em (8):

(8) Act (A) or Locution

He said to me 'Shoot her !' meaning by 'shoot' shoot and referring by 'her' to her.

Act (B) or Illocution

He urged (or advised, ordered, \&c.) me to shoot her.

Act (C.a) or Perlocution

He persuaded me to shoot her.

Act (C.b)

He got me to (or made me, etc.) shoot her (AUSTIN, 1962, p. 101-102). ${ }^{16}$

Grice ou Austin? Não importa qual teórico tenha sido o desencadeador das maiores discussões sobre questões pragmáticas no mundo linguístico. O que se sabe é que, a partir deles, expressivos nomes da Filosofia, da Lógica e da Linguística, como Stalnaker (1970), Keenan (1971), Kripke (1977) Sadock (1978), Donnellan (1978), Karttunen e Peters (1979), Gazdar (1979), Bach e Harnish (1979), Sperber e Wilson (1986, 1995) e Levinson (1983, 2000), Brown e Levinson (1987), para citar alguns, discutiram fenômenos ditos pragmáticos em seus textos, que, por sua vez, originaram um número expressivo de estudos da pragmática e suas aplicações em nível de interfaces. E todos contribuíram, de alguma maneira, para a divisão da pragmática na maneira como ela pode ser classificada. ${ }^{17}$

\section{Pragmática Near-side $\times$ Pragmática Far-side}

De acordo com Korta e Perry (2015), Pragmática Near-side preocupa-se com a natureza de certos fatos que são relevantes para determinar o que é dito. Pragmática Far-side se foca no que acontece além do dito: quais

\footnotetext{
15 Se eu prometer que estarei em casa para o jantar e mais tarde enuncio que estarei em casa para jantar, minhas ações são exemplos do mesmo ato locucionário: ambos envolvem o conteúdo de que estarei em casa para jantar. Contudo, minhas ações são instâncias de diferentes atos ilocucionários: uma tem a força de promessa, enquanto que o outro tem a força de um enunciado.

16 Ato (A) ou locução Ele me disse 'atira nela'! significando 'atirar' atirar e referindo 'ela' por ela. Ato (B) ou ilocução Ele me pressionou (ou aconselhou, ordenou, etc.) a atirar nela. Ato (C.a) ou perlocução Ele me persuadiu a atirar nela. Ato C.b) Ele me fez ( conseguiu) atirar nela.

17 Como se trata de um texto cujo propósito é fazer apenas um breve percurso teórico sobre Pragmática, nem todos os autores citados serão explicitados. Servem, no entanto, como referências para futuros trabalhos.
}

atos de fala entram em ação, ao se dizer o que se diz, ou quais implicaturas são geradas pelo o que se diz. Semântica e Pragmática trabalham com significado, mas é a Pragmática que deve explicar a informação que alguém transmite, e as ações que alguém realiza ao dizer algo.

Embora problemas com indexicais, ou dêiticos ${ }^{18}$ tenham sido abordados intensamente no desenvolvimento de lógica temporal, modal, deôntica, trabalhos como o de Montague (1968) em seu ensaio "Pragmatics" (1968), Stalnaker (1970/1999) com seu "Pragmatics", e Kaplan (1989) com seu trabalho "Demonstratives" discutiram tópicos classificados no que chamamos de Pragmática near-side, ou seja, a tentativa de resolução de fenômenos como ambiguidade e vaguidade, referência dos nomes próprios, anáfora, pressuposição em alguns aspectos e indexicais e demonstrativos.

Montague generalizou o conceito de Mundos Possíveis para lidar com um grande número de fenômenos linguísticos, entre eles, os indexicais. De acordo com o autor, indexicais combinam um mundo possível com fatores relevantes ao valor de verdade da sentença.Se temos os exemplos como (...) abaixo,

\section{(9) Fátima foi ao supermercado.}

(10) Eu fui ao supermercado.

a sentença (9) será verdadeira num mundo, em um tempo específico; enquanto que (10) será verdadeira somente se o falante foi ao supermercado em um tempo anterior a seu enunciado (anterior no mundo).

Stalnaker (1970/1999) propõe que as regras sintáticas e semânticas de uma língua determinam uma sentença interpretada, que corresponde a uma função de contextos em proposições, e uma proposição corresponde a um função de mundos possíveis a valores de verdade. $\mathrm{O}$ fenômeno pragmático que tem em mente envolve fatores contextuais na linguagem natural que interagem com o significado convencional para determinar o que é dito. Conforme Korta e Perry (2015), ao usar teoria pragmática para lidar com tópicos que normalmente seriam considerados semânticos, como existenciais negativos (Homero não existe) e informação em sentenças de identidade (A estrela da manhã é a estrela da tarde) Stalnaker continua uma tradição griceana. Em suas palavras:

I think the general Gricean strategy of trying to reduce the burden on semantics by explaining as much of the phenomena as possible in terms of truisms about

\footnotetext{
18 Interessante observar que em estudos de primeira e segunda língua, como os trabalhos pioneiros de Klein e Perdue (1993) e Tomlin (1990), concluíram que crianças e aprendizes de segunda língua que aprendem a língua alvo sem instrução formal adquirem os pronomes dêiticos eu e você $(I, y o u)$, bem antes dos que os anafóricos ele, ela (he, she, it).
} 
conversation as a rational activity remains as fruitful and promising as it was when he first proposed it (STALNAKER, 1999, p. 113). ${ }^{19}$

Por fim, ainda que Kaplan (1989) não tenha considerado sua teoria uma teoria dos enunciados ou uma teoria pragmática e, sim, uma teoria semântica dos indexicais e demonstrativos, a sua noção quádrupla de contexto de um agente, localização, tempo e mundo intuitivamente reflete o falante de um enunciado, tempo e local do enunciado e o mundo possível em que ele ocorre.

Além de Grice e Austin/Searle,dois exemplos de Pragmática far-side são aqui considerados: a Teoria da Relevância (Sperber e Wilson) e a Teoria das Implicaturas Conversacionais Generalizadas (Levinson). Apesar de ambas poderem ser vinculadas aos insights de Grice, traçam caminhos diferenciados.

Sperber e Wilson (1986, 1995), na esteira de Grice, defendem, há mais de trinta anos, uma bem sucedida abordagem sobre a interface comunicação-cognição conhecida como Teoria da Relevância (TR). Para eles, a cognição humana é dirigida por princípios fundamentais que tornam a interatividade possível e razoável, e dois são os princípios básicos que sustentam a arquitetura conceitual da TR em sua forma clássica:

1. Princípio Cognitivo de Relevância - A comunicação humana tende a ser dirigida para a maximização da relevância

2. Princípio Comunicativo de Relevância - Todo estímulo ostensivo (intenção informativa e comunicativa) comunica a presunção de sua própria relevância ótima

O estímulo é relevante o suficiente para merecer o esforço de processamento da audiência e é o mais relevante compatível com as habilidades e preferências do comunicador. O grau de relevância é diretamente proporcional à relação entre esforço de processamento e efeito cognitivo positivo. Em contextos idênticos, tanto menor o primeiro e tanto maior o segundo, mais relevante o estímulo. Além disso, Sperber e Wilson,

a) implicitamente assumem que o processo de compreensão inferencial é não demonstrativo, ou seja, até mesmo sob as melhores condições, a comunicação pode falhar, uma vez que o ouvinte não pode nem decodificar, nem deduzir a intenção comunicativa do comunicador e, sim, apenas, construir uma suposição baseada na evidência fornecida pelo comportamento ostensivo do comunicador;

\footnotetext{
${ }^{19}$ Penso que a estratégia griceana de tentar reduzir o peso da semântica, explicando tanto quanto possível os fenômenos em termos de truísmos sobre a conversação como uma atividade racional pemanece tão frutífera e promissora xomo quando ele a propôs pela primeira vez.
}

b) explicitamente assumem que qualquer informação disponível para o ouvinte, representada conceitualmente, pode ser usada como uma premissa no processo inferencial; isto é, o processo de compreensão inferencial é global oposto a local, no qual o processo local ou é livre de contexto ou sensível somente a informação contextual de um domínio de um conjunto, enquanto que o processo global tem livre acesso a todas informações conceptuais na memória. ${ }^{20}$

O trabalho de Levinson (2000), por sua vez, é um bom representante da chamada pragmática orientada pela gramática. Embora seu trabalho seja definido como neogriceano, Levinson não se compromete com a divisão griceana do que é dito e o que é implicado, mas assume princípios conversacionais e máximas, formulando uma teoria de

Implicaturas Generalizadas (GCIs), propondo uma série de heurísticas inspiradas nas máximas de Grice. Sua teoria não é filosófica nem psicológica, mas uma teoria de significado de enunciado-tipo, com foco na linguística. Conforme Levinson:

In the composite theory of meaning, the theory of GCIs plays just a small role in a general theory of communication... It is just to linguistic theory that GCIs have an unparalleled import (LEVINSON 2000, p. 21-22). ${ }^{21}$

$\mathrm{O}$ autor argumenta que o conteúdo de um enunciado consiste num nível de significado codificado ( significado da sentença), um nível de significado inferencial (enunciado-token) e um nível intermediário que não é baseado na computação direta das intenções dos falantes, mas nas expectativas gerais sobre como a língua é normalmente usada.

Três são as heurísticas estabelecidas por Levinson:

- Primeira Heurística (Q): O que não é dito não é dito;

- Segunda Heurística (I): O que é expresso simplesmente é exemplificado de maneira estereótipa;

- Terceira Heurística (M): O que é dito de forma anormal, não é normal (uma mensagem marcada indica uma situação marcada).

As três heurísticas se relacionam com as máximas de Grice e, quando em conflito, Q derrota $\mathrm{M}$ e $\mathrm{M}$ derrota I.

Isto posto, resta apenas, para o propósito deste artigo, apresentar o Quadro 2 com o resumo dos autores citados neste segundo período.

\footnotetext{
${ }^{20}$ Cf. Ibaños, Ana. 2008

${ }^{21} \mathrm{Na}$ teoria composta do significado, a Teoria das GCIs tem apenas um pequeno papel na teoria geral da comunicação.Somente para a Linguística a teoria tem um papel de importância inédita.
} 
Quadro 2. Período contemporâneo da história Pragmática

\begin{tabular}{|l|l|l|}
\hline Grice & Teoria das Implicaturas & $\begin{array}{l}\text { Investigação lógico- } \\
\text { Inguística }\end{array}$ \\
\hline Austin & Atos de fala & Pragmática do uso \\
\hline Searle & Atos de fala & Pragmática do uso \\
\hline Sperber e Wilson & Teoria da Relevância & Pragmática cognitiva \\
\hline Levinson & $\begin{array}{l}\text { Teoria de Implicaturas } \\
\text { Generalizadas }\end{array}$ & Pragmática linguística \\
\hline
\end{tabular}

\section{Últimas palavras}

As variações em torno de conceitos de pragmáticas dependem das direção e das interfaces que as diversas pragmáticas perseguem, e o objetivo deste artigo é apenas de familiarizar o leitor com conceitos que se entrecruzam numa interface problemática, mas que formam o caminho que as ciências da linguagem e da linguística tomam diante da situação da ciência do uso.

\section{Referências}

ALLWOOD, Jens. A bird's eye view of pragmatics. In GREGERSEN, Kirsten; MEY, Jacob (Ed.). Papers from the forth Scandinavian Conference of Linguistics. Hindsgavl: Odense University Press, 1978.

AUSTIN, Paul. How to do things with words. Oxford: Oxford University Press, 1962.

BAR-HILLEL, Y. Indexical Expressions. Mind, v. 63, p. 359379, 1954.

BROWN, P.; LEVINSON, S. Politeness: some universals in language use. Cambridge: Cambridge University Press, 1987.

IBAÑOS, Ana. Relevância, cognição, inferência e processos dedutivos. In: CAMPOS, Jorge; RAUEN, Fábio José (Org.). Tópicos em teoria da relevância [recurso eletrônico]. Porto Alegre: EDIPUCRS, 2008.

CARNAP, R. Introduction to Semantics. Cambridge, Mass.: MIT Press, 1942.

COSTA, Jorge Campos da. A relevância da pragmática na pragmática da relevância. Porto Alegre: EDIPUCRS, 2008.

COSTA, Jorge Campos da. A Teoria Inferencial das Implicaturas: descrição do modelo clássico de Grice. Letras de Hoje, Porto Alegre, v. 44, n. 3, p. 12-17, jul./set. 2009.

CRESSWELL, Maxwell. Logics and languages. Methuen: London, 1973.

CUMMINGS, Louise (Ed.). The pragmatics encyclopedia. London: Routledge, 2010.

DONELLAN, K. S. Speaker reference, descriptions and anaphora. In: COLE (Ed.). Syntax and semantics. Vol. 9: Pragmatics. New York: Academic Press, 1978.

FREGE, G. Lógica e filosofia da linguagem. São Paulo: Cultrix, 1978.
GAZDAR, G. English as a context-free language. University of Sussex, 1979.

GRICE, Paul. Meaning. The Philosophical Review, v. 66, 1957. (Reimpresso em: GRICE, P. Studies in the ways of words. Cambridge, Mass.: Harvard University Press, 1989. p. 213-223).

KARTTUNEN, Lauri; STANLEY, Peters. Conventional Implicature. In: OH, C. K.; DINNEN, D. (Ed.). Syntax and semantics. Vol. 11: Presupposition. New York: Academic Press, 1979.

KEENAN, E. L. Two Kinds of Presupposition in Natural Language. In: FILLMORE, C. J.; LANGENDOEN, D. T. (Ed.). Studies in linguistic semantics. New York: Holt, Rinehart \& Winston, 1971. p. 45-54.

KLEIN, Wolfgang; PERDUE, Clive. Adult language acquisition: cross-linguistic perspective. Vol. II: Results. Cambridge: Cambridge University Press, 1993.

KORTA, Kepa; PERRY, John. Pragmatics. The Stanford Encyclopedia of Philosophy. Winter 2015. Edward N. Zalta (Ed.). Available in: <https://plato.stanford.edu/archives/ win2015/entries/pragmatics/>. Acessado em: 29 maio 2017.

KRIPKE, Saul. Speaker's Reference and semantic reference. In: FRENCH, P. A.; UEHLING JR., T. T.; WETTSTEIN, H. (Ed.). Contemporary Perspectives in the Philosophy of Language. Minneapolis: University of Minnesota Press, 1977.

LEVINSON, S. Pragmatics. Cambridge: Cambridge University Press, 1983.

LEVINSON, S. Presumptive meanings. Cambridge: MIT Press, 2000.

LEWIS, David. General Semantics. Synthese, v. 22, 1970.

MONTAGUE, Richard. (1968). Pragmatics. In: THOMASON, R.H. (Ed.). Formal Philosophy. New Haven: Yale University Press, 1974.

MORRIS, Charles (1938). Foundations of the Theory of Signs. In: MORRIS. Writings on the General Theory of Signs. The Hague: Mouton, 1971.

MORRIS, Charles. Signs, language and behavior. New York: Prentice Hall, 1946.

PERNA, Cristina; GOLDNADEL, Marcos; MOLSING, Karina. Pragmáticas - vertentes contemporâneas. Porto Alegre: EDIPUCRS, 2016.

PULLUM, G. K.; GAZDAR, G. Natural languages and contextfree languages. Linguistics and Philosophy, v. 4, p.471-504, 1982.

RUSSELL, B. On Denoting. Mind, v. 14, p. 479-493, 1905.

SADOCK, J. M. On Testing for conversational implicature. In: COLE, P. Syntax and semantics: pragmatics. New York: Academic Press, 1978. p. 281-298.

SEARLE, J. Speech Acts: an essay in the philosophy of language. Cambridge: Cambridge University Press, 1969.

SEARLE, J. (1975). A taxonomy of speech acts. In: SEARLE, J.R. Expression and meaning. Cambridge: Cambridge University Press, 1979. p. 1-29.

SEARLE, J. Intentionality: an essay in the philosophy of mind. Cambridge: Cambridge University Press, 1983. 
SPERBER, Dan; WILSON, Deirdre. (1986). Relevance: communication and cognition. 2. ed. Oxford: Blackwell, 1995.

STALNAKER, Richard. Pragmatics. Synthese, v. 22, 1970.

STALNAKER, Richard. Context and Content. Oxford: Oxford University Press, 1999.

STRAWSON, P. F. Meaning and truth. Oxford: Oxford University Press, 1969.

STRAWSON, P. F. Review of Paul Grice Studies in the Way of Words. Synthese, v. 84, p. 153-161, 1990.
TOMLIN, Russell. Functionalism in second language acquisition. Studies in second language acquisition, v. 12, p. $155-177,1990$.

WITTGENSTEIN, Ludwig. Philosophical Investigations. P. M. S. Hacker and Joachim Schulte (eds. and trans.). 4.ed. Oxford: Wiley-Blackwell, 2009.

Recebido: 22 de abril de 2017

Aprovado: 24 de junho de 2017

Contato:

Ana Maria T. Ibaños < atibanos@pucrs.br>

Jorge Campos da Costa $<$ jcampos@pucrs.br> 